\title{
Fading channel emulation for massive MIMO testing using a conductive phase matrix setup
}

\author{
Pekka Kyösti ${ }^{12}$, Petteri Heino ${ }^{1}$ \\ ${ }^{1}$ Keysight Technologies Finland oy, Oulu, Finland, firstname.lastname@keysight.com \\ ${ }^{2}$ University of Oulu, Oulu, Finland, firstname.lastname@oulu.fi
}

\begin{abstract}
Functionalities, algorithms, and performances of massive MIMO base stations should be tested in versatile fading radio channel conditions. Base stations of 5G "New radio" that operate on sub $6 \mathrm{GHz}$ frequency bands typically provide antenna connectors enabling RF cable connection of test devices to the device under test (DUT). Furthermore, the number of DUT antennas is high and consequently the need of fading channel emulator (CE) resources becomes high. An approach can be taken to reduce the number of independent fading channels to be emulated. This can be done by using a phase shifting and combining unit (aka phase matrix unit) in between the DUT and CE. The phase matrix concept in fading emulation, together with its capabilities and limitations, is discussed in this paper.
\end{abstract}

Index Terms - antennas, electormagnetides, propagation, measurements.

\section{INTRODUCTION}

$5 \mathrm{G}$ networks operating at frequency range 1 (FR1), e.g. around 2.6 or $3.5 \mathrm{GHz}$, are to be deployed soon. Base stations will have in the first phase, e.g., 64 radio frequency (RF) transceiver chains, 32 for an array of one polarization and other 32 for an array of orthogonal polarization. The intention is to serve simultaneously multiple user equipment (UE) with multiple spatial streams (=layers) possibly using same time and frequency resources. For a successful communication the base station, together with UEs, must adapt and operate in time-variant frequency and space selective fading radio channel condition where the support of multiple streams heavily rely on polarization. Thus, testing of base stations in realistic multi-dimensional fading conditions is crucial in development of products and deployment of networks.

As mentioned in the abstract, FR1 base stations typically provide antenna RF connectors and traditional conducted fading emulation is possible and over-the-air testing is not mandatory. Furthermore, base station antennas, either individual elements or groups of elements coupled with fixed analogous weights, are time invariant and can be accurately obtained either by electro-magnetic simulators or by measurements. Radiation patterns can be embedded to the channel model by the standard approach of geometry based channel models as specified, e.g., in [1] or [2].

A straightforward fading test setup is to connect each RF port of the base station to be tested to a separate input/output port of a fading channel emulator (CE). The fading emulator then provides independently fading channel from each $\mathrm{BS}$ port to each UE port. This configuration is described in Section II. It is the most versatile configuration but requires a high number of fading emulator resources. The phase matrix setup concept, that provides reduced $\mathrm{CE}$ resource consumption, is described in Section III. The purpose of this work is to discuss the use of phase matrix concept and its capabilities.

\section{A. Space/angle domain analogies with frequency/delay domain}

In this sub-section we revisit a few fundamentals of stochastic radio channel. This is done to set background for the trade-offs discussed in Section III.

It is well known that a channel frequency response is the Fourier transform pair with channel impulse response [3]. The frequency domain and (time) delay domain representations are coupled by Fourier transform and both contain the same information. Analogously, space and angle are a Fourier transform pair.

Any single (delay) tap channel is flat fading in frequency. Multiple tap channels are frequency selective fading and provide frequency diversity for wideband transceivers, but a narrowband transceiver cannot utilize it. Analogously, a single direction channel is flat fading in space. Multiple direction, i.e. directionally dispersed, channels are spatially selective fading and provide space diversity for antenna arrays, but a single isotropic antenna cannot utilize it.

Delay spread and power delay profile of a channel determine the coherence bandwidth and frequency correlation function of the channel, respectively. Analogously, angular spread and power angular distribution determine the coherence distance the and the spatial correlation function of the channel.

\section{TRADITIONAL MIMO CHANNEL EMULATION}

A communication system has $M$ transmitter and $N$ receiver antennas. The system function

$$
\mathbf{Y}(t, f)=\mathbf{H}(t, f) \mathbf{X}(t, f)+\mathbf{N}(t, f)
$$

is composed of transmitted signal vector $\mathbf{X}(t, f) \in \mathbb{C}^{M \times 1}$, received signal vector $\mathbf{Y}(t, f) \in \mathbb{C}^{N \times 1}$, and a radio channel specified by a matrix format time variant transfer function $\mathbf{H}(t, f) \in \mathbb{C}^{N \times M}$. The additive noise term $\mathbf{N}(t, f) \in \mathbb{C}^{N \times 1}$ is ignored in the following. The transfer function 
$\mathbf{H}(t, f)=$

$\iint \mathbf{G}_{r x}\left(\Omega_{2}, t, f\right) \mathbf{h}\left(\Omega_{1}, \Omega_{2}, t, f\right) \mathbf{G}_{t x}^{T}\left(\Omega_{1}, t, f\right) d \Omega_{1} d \Omega_{2}$

is composed of $2 \times 2$ propagation channel coefficients $\mathbf{h}$ together with $\mathrm{Rx}$ and $\mathrm{Tx}$ antenna pattern matrices $\mathbf{G}_{r x} \in$ $\mathbb{C}^{N \times 2}$ and $\mathbf{G}_{t x} \in \mathbb{C}^{M \times 2}$, respectively. Tx can be, e.g., a single UE or several UEs with single or multiple antennas. Rx can be, e.g., a massive MIMO base station with high number of antenna elements. In the conductive emulation both $\mathrm{Tx}$ and $\mathrm{Rx}$ antennas are modelled mathematically within the channel transfer function and the physical antennas are bypassed in the connection.

The communication system can be tested with the setup of Figure 1 comprising $\mathrm{Tx}, \mathrm{Rx}$, and a fading radio channel emulator. Both $\mathrm{Tx}$ and $\mathrm{Rx}$ are connected to the fading emulator with, e.g., coaxial cables. In the full antenna array sampling case the fading emulator provides full $N \times M$ channel matrix, with all cross-channels included, between the linkends. If the number of antenna elements $N$ and $M$ is high, the overall need of fading channels and thus fading emulators hardware resources becomes high as well. For example, a configuration of $64 \times 8$ emulation setup is practically implementable for high accuracy link performance evaluations. The need of fading emulator resources may grow prohibitive high if, e.g., handover emulations with multiple base stations are targeted.

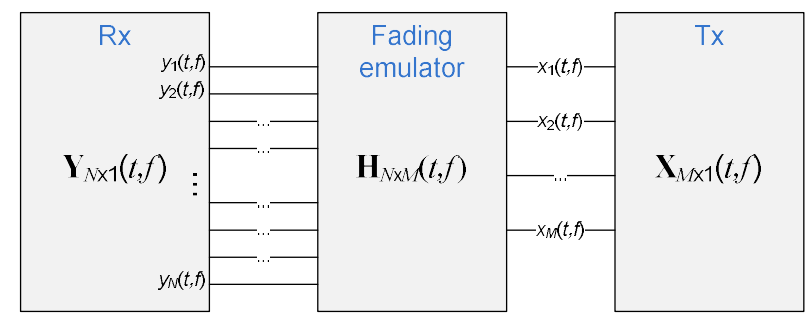

Figure 1. Full conductive test setup for MIMO fading testing.

\section{PARTIAL SAMPLING SETUP WITH PHASE MATRIX OPERATION}

The amount of fading emulation resources, i.e., the number of $\mathrm{CE}$ input/output ports and independent fading channels, can be reduced by introducing a phase shifting and combining unit before (or after) the fading emulator, as shown in Figure 2. This comes with a cost of reduced accuracy of spatial fading emulation. The phase matrix unit combines $N$ signals to $K$ signals, and the overall number of fading channels is reduced from $N M$ to $K M$. Each fading channel implements time variant frequency selective fading with multiple delayed propagation paths. The new system function is

$$
\mathbf{Y}(t, f)=\mathbf{F}(t, f) \mathbf{W}(t, f) \mathbf{X}(t, f)
$$

where $\mathbf{F}(t, f) \in \mathbb{C}^{N \times K}$ is the phase shifting and coupling matrix, and $\mathbf{W}(t, f) \in \mathbb{C}^{K \times M}$ is a modified MIMO channel matrix within the fading emulator. The phase matrix $\mathbf{F}(t, f)$ contains the combing weights. In a practical case, the phase matrix is time and frequency invariant and thus $\mathbf{F}(t, f)=\mathbf{F}$.

UE (Tx) antenna characteristics are embedded to the transfer matrix $\mathbf{W}$ and implemented by the fading emulator, similarly to the full sampling case of Section 2. The role of phase matrix unit is only to reduce the degrees of freedom. Temporal/Doppler, frequency/delay, and polarization phenomena, as well as all UE side effects are implemented by the fading emulator.

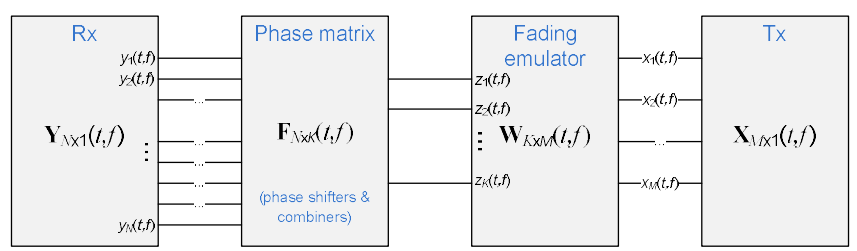

Figure 2. A partial sampling MIMO fading test setup with a phase shifting and combining operation.

The partial sampling setup reduces spatial multi-path richness of the emulated channel model. For example, the setup provides a single (spatial) fading path per link if $K=$ $M$ and there are $M$ single antenna UEs. With clean (pure) LOS channel model there is only one path per link (neglecting polarization dimension) and the phase matrix setup can be used without loss of accuracy or generality. If the target channel model has only few significant (spatial) multi-paths, only small accuracy loss is experienced even when $K$ is only modestly higher than $M$. On the other hand, in a multi-path rich NLOS channel model the use of phase matrix to reduce $\mathrm{CE}$ resources may cause notable accuracy losses and deviations from the target channel model.

\section{Phase MATRIX USE CASES}

There are several ways of using and interpreting the role of the phase matrix. In the following one of them is described.

\section{A. Virtual multi-probe OTA setup}

In Figure 3 is illustrated an interpretation that follows analogy with a multi-probe over-the-air (OTA) test setup. In total $K$ fictitious probes, i.e. test antennas, are placed in a fictitious OTA chamber. The probes are characterized by their direction (space angle) and their polarization. The polarization definition is in the same coordinate system with Tx and Rx antennas characteristics. The probe characteristics, especially their number and directions can be optimized based on the target channel model. Geometric clusters of a target model as observable by the device under test (Rx) are typically approximated by discrete probe locations in the regular OTA emulation. One or several probes can be used to construct a single cluster.

The phase matrix is now essentially composed of the following (DFT) beamforming weights 


$$
\begin{aligned}
\mathbf{B}= & \left\{e^{-j\left(\boldsymbol{\beta}_{k} \cdot \mathbf{r}_{n}\right)}\right\}_{n=1, \ldots, N, k=1, \ldots, K}= \\
& {\left[\begin{array}{ccc}
e^{-j\left(\boldsymbol{\beta}_{1} \cdot \mathbf{r}_{1}\right)} & \cdots & e^{-j\left(\boldsymbol{\beta}_{K} \cdot \mathbf{r}_{1}\right)} \\
\vdots & \ddots & \vdots \\
e^{-j\left(\boldsymbol{\beta}_{1} \cdot \mathbf{r}_{N}\right)} & \cdots & e^{-j\left(\boldsymbol{\beta}_{K} \cdot \mathbf{r}_{N}\right)}
\end{array}\right], }
\end{aligned}
$$

where $\cdot$ is the vector dot product operator, $\boldsymbol{\beta}_{k}$ is the wave vector from direction of the $k$ th fictitious probe, and $\mathbf{r}_{n}$ is the location vector of the $n$th $\mathrm{Rx}$ antenna. An extra term can be multiplied to the phase matrix if the $\mathrm{Rx}$ antenna model $\mathbf{G}_{r x}$ and the fictitious probe gain matrix $\mathbf{G}_{o} \in \mathbb{C}^{K \times 2}$ are considered. The term is

$$
g_{n k}=\left|\boldsymbol{G}_{r x, n}\left(\Omega_{k}\right) \boldsymbol{G}_{o, k}^{T}\right|,
$$

where $\mathbf{G}_{r x, n}\left(\Omega_{k}\right)$ denotes the $n$th row of $\mathrm{Rx}$ antenna pattern matrix to direction of $k$ th fictitious probe and $\mathbf{G}_{o, k}^{T}$ denotes the $k$ th row of the fictitious probe gain matrix.

Now the phase matrix is defined as

$$
\mathbf{F}=\left[\begin{array}{ccc}
g_{11} e^{-j\left(\boldsymbol{\beta}_{1} \cdot \mathbf{r}_{1}\right)} & \cdots & g_{1 K} e^{-j\left(\boldsymbol{\beta}_{K} \cdot \mathbf{r}_{1}\right)} \\
\vdots & \ddots & \vdots \\
g_{N 1} e^{-j\left(\boldsymbol{\beta}_{1} \cdot \mathbf{r}_{N}\right)} & \cdots & g_{N K} e^{-j\left(\boldsymbol{\beta}_{K} \cdot \mathbf{r}_{N}\right)}
\end{array}\right] .
$$

Above, the role of $\mathbf{G}_{o}$ is only to consider polarization of the probe and the effect of polarization mismatch between the probe and Rx antennas. The terms $g_{k n}$ can be ignored (i.e. set to unity) if the polarization and $\mathrm{Rx}$ antenna amplitude gains are not considered or if the phase matrix unit is capable only to phase shifting and to setting binary gain (on/off). Use of phases (beamforming weights) $\mathbf{B}$ makes the $\mathrm{Rx}$ array to observe plane waves from directions $\boldsymbol{\beta}_{k}$ of fictitious probes.

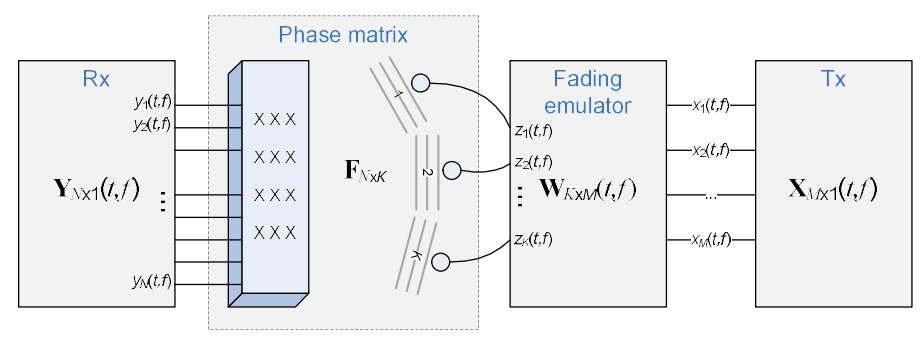

Figure 3. Illustration of the function of the phase matrix unit.

The $k$ th row of channel transfer matrix within the fading emulator in the case of a geometry based model is

$$
\begin{gathered}
\boldsymbol{W}_{\mathrm{k}}(\mathrm{t}, \mathrm{f})= \\
\sum_{\mathrm{l}=1}^{\mathrm{L}}\left[\gamma_{1, \mathrm{k}}^{\theta} \quad \gamma_{1, \mathrm{k}}^{\varphi}\right] \iint \boldsymbol{h}_{\mathrm{l}}\left(\Omega_{1}, \Omega_{2}, \mathrm{t}, \mathrm{f}\right) \boldsymbol{G}_{\mathrm{tx}}^{\mathrm{T}}\left(\Omega_{1}, \mathrm{t}, \mathrm{f}\right) \mathrm{d} \Omega_{1} \mathrm{~d} \Omega_{2},
\end{gathered}
$$

where $\gamma_{l, k}^{\theta}$ and $\gamma_{l, k}^{\varphi}$ are the weights of $k$ th fictitious probe and $l$ th path for $\theta$ and $\varphi$ polarizations, respectively. Matrix $\mathbf{h}_{l}\left(\Omega_{1}, \Omega_{2}, t, f\right)$ is the bi-directional time-variant $2 \times 2$ propagation matrix for path $l$, specified by the target channel model.
Possibly all $K M$ fading channels are not needed in the fading emulator. In other words, the channel transfer matrix $\mathbf{W}$ may be sparse. This may occur if, e.g., $M$ Tx antennas are located in multiple UEs and all UEs "are not illuminating all clusters", i.e. if the channel gain from certain of the $M$ Tx antennas to certain of the $K$ probes is negligible low. This fully depends on the weights $\gamma_{l, k}^{\theta}$ and $\gamma_{l, k}^{\varphi}$ set for the fictitious probes.

\section{SimULATION RESUlTS}

\section{A. Comparison of the reference setup and simulated phase matrix}

The base station array has 64 antennas with two orthogonal polarizations. Orthogonally polarized antennas are co-located. Thus, there are 32 different antenna locations in the array. Antennas have half wavelength spacing in horizontal domain and more than one wavelength spacing in vertical domain. The violation of Nyquist sampling in vertical domain causes aliasing in elevation domain, as can be observed in Figure 5 and Figure 5.

The reference setup is the full 64 output port fading emulator configuration, simulated from Keysight GCM Tool generated emulation files. In other words, it is the full MUMIMO channel matrix from all 64 BS antenna ports to four single antenna UEs. The target channel model is CDL-B NLOS model [2] with the strongest cluster direction oriented w.r.t. different LOS directions of four different UE locations.

The phase matrix setup has $64 \times 16$ phase shifter. It has two dual polarized virtual probes (= virtual clusters) per link. A perfect fading channel model, with same parameters as the reference model, is generated for the $16 \times 4$ fading emulation as described in Section III.A. Directions of the virtual probes are selected by taking directions of the two strongest clusters of the target channel model.
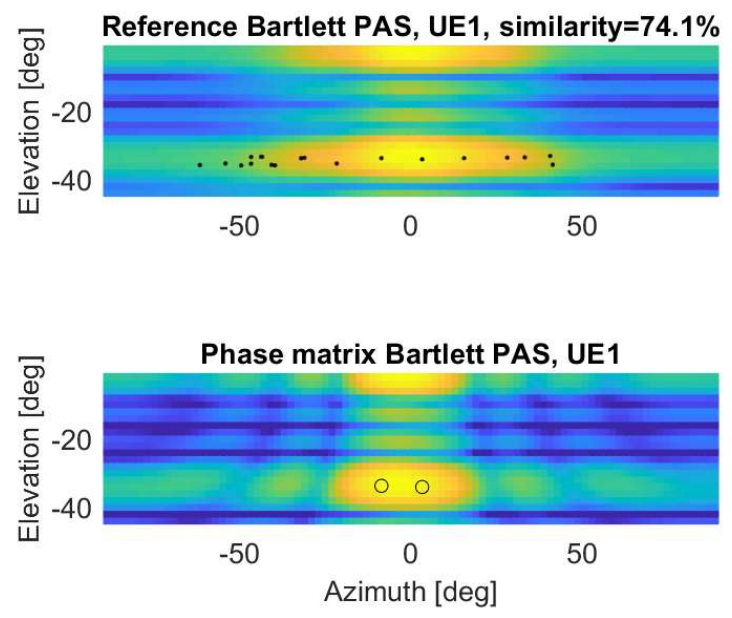

Figure 4. Bartlett PAS estimate of radio channel to UE1 for the reference model and the $64 \times 16$ phase matrix setup. 

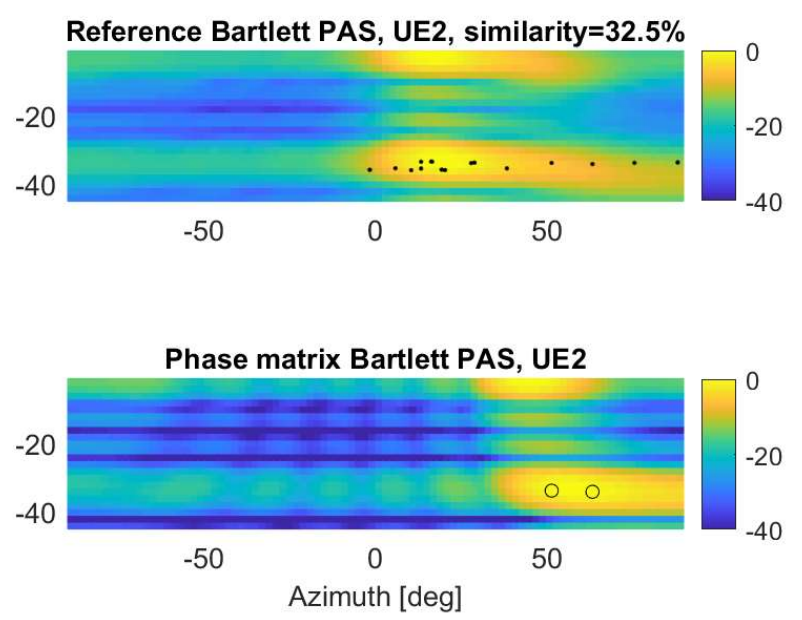

Figure 5. Bartlett PAS estimate of radio channel to UE2 for the reference model and the $64 \times 16$ phase matrix setup.

Figure 5 and Figure 5 show the estimated PAS as observed by the DUT, i.e., UE1 and UE2. On both UE, the top figure illustrates the reference Bartlett PAS and the bottom figure the one with phase matrix setup. Black dots are cluster directions of the of the target channel model. Black circles are directions of the virtual probes. Two dual polarized virtual probes per UE. We can immediately observe that UE1 case is better than UE2, since the target PAS is focused around BS broad side, i.e. zero azimuth. In UE2 case the target PAS is on the positive azimuth axis. Evidently the criteria for choosing virtual probe directions in this case was not optimal, as the phase matrix PAS deviates substantially from the reference. Further work is needed to choose optimal virtual probe directions. The problem is similar to the probe placement in design of multi-probe anechoic chamber (MPAC) OTA emulation setups.

The total variation distance of PAS is introduced in [4] as a metric for evaluating different multi-probe anechoic chamber (MPAC) based OTA fading emulation setups. The same metric can be used also to evaluate accuracy (in spatial/polarimetric domains) of different conductive fading emulation setups. In four sub-figures of Figure 5 is given similarity \%. It is $(1-$ total variation distance of $P A S) \cdot 100 \%$, where $100 \%$ indicates full similarity and $0 \%$ complete dissimilarity.

\section{DISCUSSION}

We have specified the full MIMO fading emulation setup and a reduced partial sampling setup based of a phase matrix unit that couples the DUT to a fading emulator. The introduced use of the phase matrix is so called virtual probe case. We proposed also an evaluation metric for comparing spatial accuracy of different conductive fading emulation setups. Fully faded emulation approach remains to be the most accurate method to emulate for example standard 3GPP
$5 \mathrm{G}$ channel models of [2]. Alternatives such as methods based on a phase matrix exists and may provide lower cost or scalability in some use cases and with suitable models.

Further research is required to understand applicability of the partial sampling (phase matrix) setup. New performance metric may be needed or at least acceptance limits for the proposed PAS similarity percentage must be determined. The optimal method of finding directions of virtual probes is to be investigated.

\section{ACKNOWLEDGEMENT}

This work has been partly done in 6G Flagship programme funded by Academy of Finland (grant no. 318927).

\section{REFERENCES}

[1] A. F. Molisch, "A generic model for MIMO wireless propagation channels in macro- and microcells," in IEEE Transactions on Signal Processing, vol. 52, no. 1, pp. 61-71, Jan. 2004.

[2] TR 38.901, "Study on channel model for frequencies from 0.5 to 100 GHz,”3GPP, Tech. Rep. V14.1.1, July 2017.

[3] M. Pätzold, "System function and characteristic quantities of spatial deterministic Gaussian uncorrelated scattering processes," The 57th IEEE Semi-annual Vehicular Technology Conference, 2003. VTC 2003-Spring., Jeju, South Korea, 2003, pp. 256-261 vol.1.

[4] P. Kyösti, L. Hentilä, W. Fan, J. Lehtomäki and M. Latva-Aho, "On Radiated Performance Evaluation of Massive MIMO Devices in Multiprobe Anechoic Chamber OTA Setups," in IEEE Transactions on Antennas and Propagation, vol. 66, no. 10, pp. 5485-5497, Oct. 2018.

[5] M. Young, The Technical Writer's Handbook. Mill Valley, CA: University Science, 1989 